\title{
Validación de una estrategia para la estimación del riesgo en intersecciones con vehículos conectados
}

\author{
Jorge Villagra \\ jorge.villagra@csic.es \\ Manuel Perarnau, Jorge Godoy, Antonio Artuñedo \\ \{manuel.perarnau,jorge.godoy\}@upm.es, antonio.artunedo@car.upm-csic.es
}

\section{Resumen}

Para hacer posible el despliegue masivo de vehículos automatizados en entornos urbanos es capital avanzar en la toma de decisiones seguras. En particular, es necesario mejorar la capacidad de inferir las intenciones de los diferentes agentes $y$ el riesgo que implican en las escenas complejas de conducción, mejorando así la seguridad y predictibilidad de los sistemas de ayuda a la conducción y de conducción automatizada. El presente trabajo muestra la implementación y validación en simulación de una solución novedosa para estimar el riesgo de conducción utilizando un modelo de espacio y estados en el contexto de las intersecciones. La estrategia utilizada modela la escena de conducción como una red dinámica Bayesiana e infiere intenciones y expectativas de los agentes involucrados a través de un filtro de partículas. Los resultados son muy prometedores tanto en tasa de acierto como en horizonte de predicción en los entornos para los que ha sido probado: intersecciones en $Y$, en $T$ y en $X$.

Palabras clave: Evaluación de riesgos, vehículos inteligentes, red Bayesiana dinámica, filtro de partículas

\section{INTRODUCCIÓN}

La toma de decisiones bajo importantes fuentes de incertidumbre es un problema difícil de resolver cuando se navega en condiciones de tráfico reales utilizando sensores comerciales. La comprensión de la relación espacio-temporal entre el vehículo sujeto y las entidades pertinentes del entorno navegable -red de carreteras- puede ser completamente errática, y en consecuencia, el comportamiento del sistema automatizado puede acarrear consecuencias indeseadas.

En efecto, la estimación de riesgos es una de las áreas funcionales de la conducción autónoma en la que más avances son necesarios, en especial en entornos urbanos complejos, como las intersecciones o rotondas. Este trabajo presenta la implementación y validación en simulación de una estrategia para estimar el riesgo de conducción en varios tipos de intersecciones. La Fig. 1 muestra un ejemplo de los casos evaluados y la utilidad potencial del estimador de riesgo planteado. En el vehículo rojo (V3) se estimará el riesgo potencial de cada uno de los 2 vehículos de la escena sobre el vehículo propio. El resultado, en caso de generar una alerta por su elevada peligrosidad, se podrá comunicar inalámbricamente a los vehículos implicados para que tomen las medidas oprtunas (frenado de emergancia en este caso) o se utilizará en el vehículo propio para adecuar su velocidad al contexto de riesgo al que se enfrente.

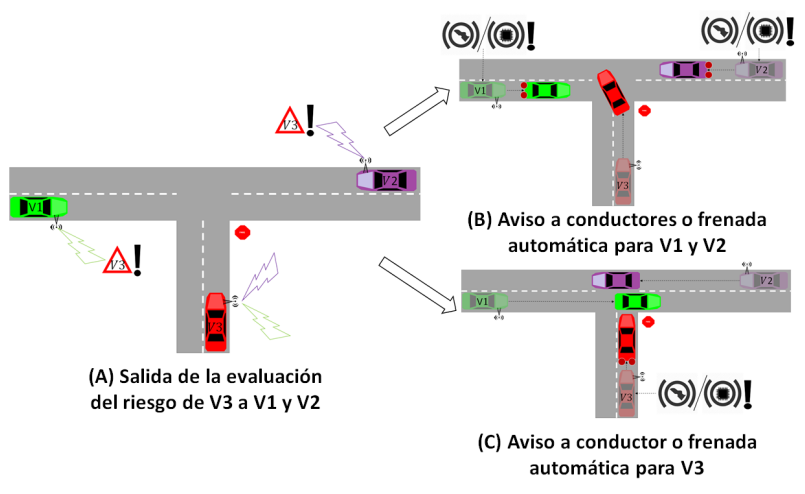

Figura 1: Salida de la información de la situación de riesgo por comunicación $\mathrm{V} 2 \mathrm{~V}$ y posibles acciones a tomar.

El artículo se estructura como sigue. En la Sección 2 se repasa el estado del arte para poder contextualizar el interés del trabajo presentado. Después, con objeto de formalizar las técnicas empleadas, se presenta sucesivamente el modelo de interacción y las técnicas de inferencia de riesgo utilizados, en las secciones 3 y 4, respectivamente. La sección 5 presenta la metodología de evaluación seguida y los resultados obtenidos, y finalmente, se dan algunas conclusiones en la sección 6 .

\section{ESTADO DEL ARTE}

Con mucho, el paradigma más popular para la estimación del riesgo de colisión en robótica es el de "Predicción de trayectoria + detección de colisiones", compuesto de dos pasos: (i) predecir las 
posibles trayectorias futuras para todas las entidades en movimiento en la escena, (ii) detectar colisiones entre pares de trayectorias, y derivar una estimación de riesgo basada en el total posibilidad de colisión.

En relación a la predicción de movimiento [7] propone una clasificación a nivel conceptual, basada en las diferentes formas de explicar el movimiento de un vehículo:

- Modelos físicos: Consideran que el movimiento de los vehículos está limitados por las leyes de la física, sin contemplar la existencia de otros vehículos. Los modelos de evolución se pueden usar para la predicción de trayectorias de varias maneras, siendo la principal diferencia entre ellos el manejo de las incertidumbres (e.g. simulación de trayectoria única [9], simulación de ruido gaussiano [10] o simulaciones de Monte Carlo [1]) El principal inconveniente de esta aproximación es que al basarse en propiedades de movimiento de bajo nivel (cinemáticas o dinámicas), los modelos de movimiento de las "entidades físicas" se limitan a la predicción de movimiento a corto plazo. Por esta misma razón, estos modelos no podrán prever ningún cambio en el movimiento del automóvil causado por la ejecución de una maniobra particular o por cambios debidos a factores externos.

- Modelos basados en maniobras: Consideran que el movimiento de los vehículos dependen de las maniobras que sus conductores tienen la intención de realizar, por lo que si se identifica la maniobra, es posible predecir el movimiento del vehículo. Se basan en el estado físico del vehículo (velocidad, posición, dirección), la información de la vía (geometría, topología, limites de velocidad, leyes de tráfico) y comportamiento del conductor (movimiento de la cabeza, estilo de conducción). Para la clasificación de maniobras en escenarios complejos se emplean algoritmos de aprendizaje como Multi Layer Peceptrons (MLP) [11], Support Vector Machines (SVM) [2] o modelos ocultos de Markov [3]. Aunque esta aproximación permite predecir la intención de un conductor mediante el ajuste de las trayectorias y estados, no tiene en cuenta las dependencias entre vehículos, algo especialmente relevante en el contexto de las intersecciones, donde las reglas de prioridad se han de considerar para juzgar la maniobra de un vehículo

- Modelos basados en interacciones: Se basan en el principio de que para realizar una determinada maniobra, los vehículos reconocen su mutua presencia, lo que, en comparación con los modelos anteriores, permite mejorar el entendimiento del contexto de la situación. Para la generación de trayectorias no seria posible considerar todas las dependencias entre vehículos ya que de lo contrario el numero de casos seria inviable. Sin embargo llegado el momento de comparar la trayectoria actual con las registradas, es posible basarse en el simple hecho de seleccionar las dependencias cuyas trayectorias impongan el menor riesgo de colisión. Para resolver este problema han propuesto en el pasado modelos de Markov ocultos y acoplados [5], pero su coste computacional es inasumible. Para simplificar el problema alguno trabajos consideran únicamente la influencia del vehículo de interés que influiría de manera directa en la maniobra de otro. Dicha simplificación se puede usar en situaciones de cambio de carril, adelantamientos [13], seguimiento de otros vehículos [8].

El trabajo de [6] es una propuesta innovadora dentro de los modelos basados en interacciones, pues permite resolver la inferencia de riesgo teniendo en cuenta la incertidumbre, la topología y el contexto de la situación según las normas de tráfico. Sin embargo, la validación de este prometedor enfoque se hizo sólo teniendo en cuenta 2 vehículos y en un caso muy concreto: la intersección no señalizada en T. Hasta donde sabemos, esta es la primera contribución en la literatura que, apoyándose en las técnicas de estimación de riesgo basadas en la interacción de los agentes de conducción, permite cuantificar el dominio de validez de la estrategia en (i) en diferentes tipos de intersecciones y (ii) en escenarios con más de 2 vehículos.

\section{FORMULACIÓN DEL MODELO}

El modelo usado en el presente se apoya en el formalismo de los Modelos de Markov de Espacio y Estado (MSSMs). Esta aproximación es un tipo de red dinámica Bayesiana que describe la dinámica de la escena usando variables de estado, funciones de probabilidad y medidas. Para evitar la complejidad de los conjuntos de modelos ocultos de Markov y su importante coste computacional asociado, se considera una única que permita comparar la maniobra esperada, según las leyes de trafico y la interacción con otros vehículos, con las intenciones del conductor (ver Fig. 2). Según el modelo general de la figura 2, en el que se definen las siguientes variables 


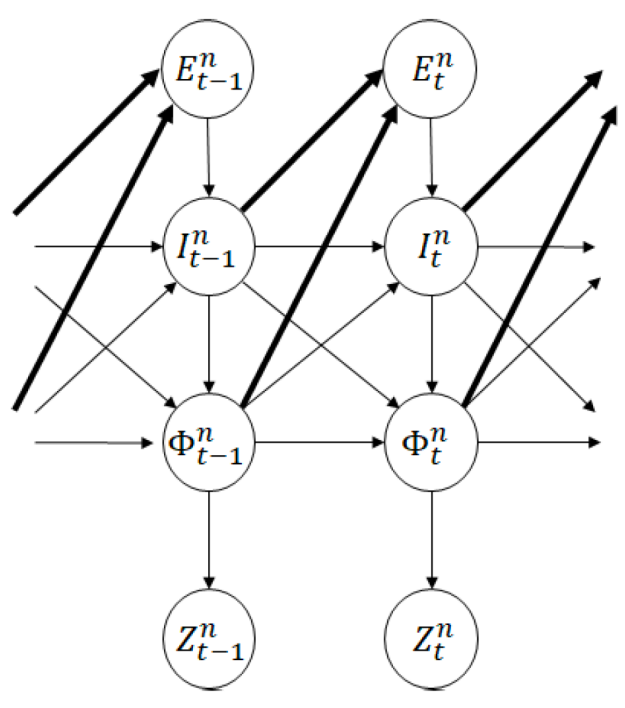

Figura 2: Estructura de la Red Dinámica Bayesiana utilizada

- $\mathbf{E}_{\mathbf{t}}^{\mathbf{n}}$ : Maniobra longitudinal esperada

- $\mathbf{I}_{\mathbf{t}}^{\mathbf{n}}\left(\mathbf{s}_{\mathbf{t}}^{\mathbf{n}}, \mathbf{I} \mathbf{c}_{\mathbf{t}}^{\mathbf{n}}\right)$ : Maniobra intencionada (longitudinal y lateral)

- $\boldsymbol{\Phi}_{\mathbf{t}}^{\mathbf{n}}\left(\mathbf{P}_{\mathbf{t}}^{\mathbf{n}}, \mathbf{S}_{\mathbf{t}}^{\mathbf{n}}\right)$ : Estado físico (posición y velocidad del vehículo)

- $\mathbf{Z}_{\mathbf{t}}^{\mathbf{n}}\left(\mathbf{P m}_{\mathbf{t}}^{\mathbf{n}}, \mathbf{S m}_{\mathbf{t}}^{\mathbf{n}}\right.$ ): Medidas (posición y velocidad del vehículo)

se extrae la siguiente distribución generalizada

$P\left(\mathbf{E}_{\mathbf{0}: \mathbf{T}}, \mathbf{I}_{\mathbf{0}: \mathbf{T}}, \boldsymbol{\Phi}_{\mathbf{0}: \mathbf{T}}, \mathbf{Z}_{\mathbf{0}: \mathbf{T}}\right)=P\left(\mathbf{E}_{\mathbf{0}}, \mathbf{I}_{\mathbf{0}}, \boldsymbol{\Phi}_{\mathbf{0}}, \mathbf{Z}_{\mathbf{0}}\right) \times$

$\times \prod_{t=1}^{T} \times \prod_{n=1}^{N}\left[P\left(E_{t}^{n} \mid \mathbf{I}_{\mathbf{t}-\mathbf{1}} \mathbf{\Phi}_{\mathbf{t}-\mathbf{1}}\right) \times P\left(I_{t}^{n} \mid \Phi_{t-1}^{n} I_{t-1}^{n} E_{1}^{n}\right)\right.$

$\left.\times P\left(\Phi_{t}^{n} \mid \Phi_{t-1}^{n} I_{t-1}^{n} I_{t}^{n}\right) \times P\left(Z_{t}^{n} \mid \Phi_{t}^{n}\right)\right]$

donde las variables en negrita indican las dependencias del vehículo $n$ respecto a los demás.

Este modelo genérico adquiere, en el caso de las intersecciones, la siguiente forma

$$
\begin{aligned}
& P\left(\mathbf{E}_{\mathbf{0}: \mathbf{T}}, \mathbf{I}_{\mathbf{0 : T}}, \mathbf{\Phi}_{\mathbf{0}: \mathbf{T}}, \mathbf{Z}_{\mathbf{0 : \mathbf { T }}}\right)=P\left(\mathbf{E}_{\mathbf{0}}, \mathbf{I}_{\mathbf{0}}, \mathbf{\Phi}_{\mathbf{0}}, \mathbf{Z}_{\mathbf{0}}\right) \\
& \times \prod_{t=1}^{T} \times \prod_{n=1}^{N}\left[P\left(E s_{t}^{n} \mid \mathbf{I}_{\mathbf{t}-\mathbf{1}} \mathbf{P}_{\mathbf{t}-\mathbf{1}} \mathbf{S}_{\mathbf{t}-\mathbf{1}}\right)\right. \\
& \times P\left(I s_{t}^{n} \mid I s_{t-1}^{n} E s_{t}^{n}\right) \times P\left(I c_{t}^{n} \mid I c_{t-1}^{n}\right) \\
& \times P\left(P_{t}^{n} \mid P_{t-1}^{n} S_{t-1}^{n} I c_{t-1}^{n}\right) \times P\left(S_{t}^{n} \mid P_{t-1}^{n} S_{t-1}^{n} I c_{t}^{n} I s_{t}^{n}\right) \\
& \left.\times P\left(P m_{t}^{n} \mid P_{t}^{n}\right) \times P\left(S m_{t}^{n} \mid s_{t}^{n}\right)\right]
\end{aligned}
$$

pudiéndose describir las inter-relacionaes de las distintas formas paramétricas como aparece en la Fig. 3. El origen y obtención de las expresiones de cada uno de los términos que aparecen en ()1) se detallan en [6] y se resumen a continuación:
Movimiento longitudinal esperado $E s_{t}^{n}$ : Se basa en [12], según el cual la percepción del ser humano es proporcional al estimulo original, por lo que el tiempo necesario para ejecutar una maniobra no es un resultado directo de las distancias y velocidades de los vehículos involucrados y si modela como una función de distribución de probabilidades:

$$
\begin{aligned}
& P\left(E_{t}^{n}=S T O P \mid\left[\mathbf{I} \mathbf{c}_{\mathbf{t}-\mathbf{1}}=\mathbf{c}_{\mathbf{t}-\mathbf{1}}\right]\left[\mathbf{P}_{\mathbf{t}-\mathbf{1}}=\mathbf{p}_{\mathbf{t}-\mathbf{1}}\right]\right. \\
& \left.\left[\mathbf{S}_{\mathbf{t}-\mathbf{1}}=\mathbf{s}_{\mathbf{t}-\mathbf{1}}\right]\right)=f\left(\mathbf{c}_{\mathbf{t}-\mathbf{1}}, \mathbf{p}_{\mathbf{t}-\mathbf{1}}, \mathbf{s}_{\mathbf{t}-\mathbf{1}}\right), \\
& f=1.0-\frac{1}{1+e^{-\lambda\left(\ln \left(g_{\min }\right)+(1-\alpha) \ln \left(S_{t-1}^{k}\right)-\ln (\gamma)\right)}}
\end{aligned}
$$

siendo $g_{\text {min }}$ el intervalo de tiempo calculado en el paso anterior, $S_{t-1}^{k}$ la velocidad del vehículo $k$ del instante de tiempo anterior y $\lambda, \alpha$ y $\beta$ las constantes empleadas por el modelo.

Movimiento longitudinal intencionado $I s_{t}^{n}$ : La estrategia aplicada consiste en comparar la maniobra intencionada del instante anterior, con lo que se espera que el conductor debe hacer para el instante actual.

$$
P\left(I s_{t}^{n} \mid \Phi_{t-1}^{n} I_{t-1}^{n} E_{t}^{n}\right)=P\left(I s_{t}^{n} \mid I s_{t-1}^{n} E s_{t}^{n}\right)
$$

siguiendo 1 tabla lógica siguiente:

\begin{tabular}{ccc}
$\mathbf{I}_{\mathbf{t}-\mathbf{1}}^{\mathbf{n}}$ & $\mathbf{E s}_{\mathbf{t}}^{\mathbf{n}}$ & $\mathbf{P}\left(\left[\mathbf{I}_{\mathbf{t}}^{\mathbf{n}}=\mathbf{s t o p}\right] \mid \mathbf{I s}_{\mathbf{t}-\mathbf{1}}^{\mathbf{n}} \mathbf{E s}_{\mathbf{t}}^{\mathbf{n}}\right)$ \\
\hline \hline$g o$ & go & $1.0-P_{\text {comply }}$ \\
\hline go & stop & 0.5 \\
\hline stop & go & 0.5 \\
\hline stop & stop & $P_{\text {comply }}$ \\
\hline
\end{tabular}

donde $P_{\text {comply }}$ es un valor entre 0 y 1 que refleja el grado de cumplimiento de las normas del conductor.

Movimiento lateral intencionado $I c_{t}^{n}$ : Se basa en la suposición de que cuando un conductor circula por una vía, su tendencia natural será la de mantenerse en ella, o dicho de otro modo:

$$
P\left(I c_{t}^{n} \mid \Phi_{t-1}^{n} I_{t-1}^{n} E_{t}^{n}\right)=P\left(I c_{t}^{n} \mid I c_{t-1}^{n}\right)
$$

donde la probabilidad de permanecer o no en la misma ruta para el movimiento lateral se calcula:

$$
P\left(I c_{t}^{n}=c_{t}^{n} \mid I c_{t-1}^{n}=c_{t-1}^{n}\right)=\left\{\begin{array}{c}
P_{\text {same }}, \text { si } c_{t}=c_{t-1} \\
\frac{1.0-P_{s a m e}}{N_{c}-1}, \text { si } c_{t} \neq c_{t-1}
\end{array}\right.
$$

siendo $P_{\text {same }} \in[0,1]$ un parámetro dependiente depende del conductor y su tendencia a variar de ruta y $N_{c}$ el numero de rutas posibles.

Pose $P_{t}^{n}$ : Se asume que la maniobra que va a ejecutar un conductor depende de la ruta que éste vaya a seguir. Para calcular la posición del 
vehículo para el instante de tiempo actual, se emplea la intención lateral, posición y velocidad del instante anterior:

$P\left(P_{t}^{n} \mid\left[P_{t-1}^{n}=p_{t-1}^{n}\right]\left[S_{t-1}^{n}=s_{t-1}^{n}\right]\left[I c_{t-1}^{n}=c_{t-1}^{n}\right]\right)=$ $\mathcal{N}\left(\mu_{x y \theta}\left(p_{t-1}^{n}, s_{t-1}^{n}, c_{t-1}^{n}\right), \sigma_{x y \theta}\right)$

donde $\mu_{x y \theta}\left(p_{t-1}^{n}, s_{t-1}^{n}, c_{t-1}^{n}\right)$ es la pose promedio que contiene $\left(\mu_{x}, \mu_{y}, \mu_{\theta}\right)$ y $\sigma_{x y \theta}$ contiene la desviación estándar para cada termino $\left(\sigma_{x}, \sigma_{y}, \sigma_{\theta}\right)$

Velocidad $S_{t}^{n}$ : La distribución de la velocidad se asume normal y esta definida por:

$P\left(S_{t}^{n} \mid\left[P_{t-1}^{n}=p_{t-1}^{n}\right]\left[S_{t-1}^{n}=s_{t-1}^{n}\right]\left[I c_{t}^{n}=c_{t}^{n}\right]\left[I s_{t}^{n}=s_{t}^{n}\right]\right)$ $=\mathcal{N}\left(\mu_{s}\left(p_{t-1}^{n}, s_{t-1}^{n}, c_{t}^{n}, s_{t}^{n}\right), \sigma_{s}\left(p_{t-1}^{n}, s_{t-1}^{n}, c_{t}^{n}, s_{t}^{n}\right)\right)$

donde $\mu_{s}\left(p_{t-1}^{n}, s_{t-1}^{n}, c_{t}^{n}, s_{t}^{n}\right)$ es una función que calcula la velocidad del vehículo en función de la velocidad y pose del instante anterior e intención y ruta actuales, teniendo en cuenta un modelo de evolución de velocidad con perfiles genéricos [6], y $\sigma_{s}\left(p_{t-1}^{n}, s_{t-1}^{n}, c_{t}^{n}, s_{t}^{n}\right)$ computa a partir del calculo anterior una desviación estándar para la construcción dinámica de una distribución de probabilidades de velocidad.

Pose medida $P m_{t}^{n}$ : Las incertidumbres de las predicciones de $x, y$ y $\theta$ se modelan empleando una distribución normal de probabilidades:

$$
P\left(P m_{t}^{n} \mid P_{t}^{n}=p_{t}^{n}\right)=\mathcal{N}\left(p_{t}^{n}, \sigma_{x y \theta}\right)
$$

donde $\sigma_{x y \theta}=\left(\sigma_{x}, \sigma_{y}, \sigma_{\theta}\right)$ son las desviaciones estándar para cada variable.

Velocidad medida $S m_{t}^{n}$ : Se asume que las medidas provenientes de los sensores son lo suficientemente precisas como para ignorar las incertidumbres, por lo que se emplea una función de Dirac

$$
P\left(\left[S m_{t}^{n}=s m_{t}^{n}\right] \mid\left[S_{t}^{n}=s_{t}^{n}\right]\right)=\delta\left(s_{t}^{n}-s m_{t}^{n}\right)
$$

\section{FILTRO DE PARTÍCULAS}

Para la detección de situaciones de riesgo se compara la maniobra que se espera que debería ejecutar el conductor respecto a sus intenciones en el contexto de las intersecciones:

$$
P\left(\left[I s_{t}^{n}=G O\right]\left[E s_{t}^{n}=S T O P\right] \mid \mathbf{P m}_{\mathbf{0}: \mathbf{t}} \mathbf{S m}_{\mathbf{0}: \mathbf{t}}\right)
$$

Cada uno de los posibles estados del sistema $\left(E_{t}, I_{t}, \Phi_{t}\right.$ para todos los vehículos) estará contenido en lo que llamará una partícula, que tendrá un peso asociado dependiente de su proximidad con la realidad, y que se representa como sigue:

$$
\left\{H_{i, t}, w_{i, t}\right\}_{i=1: N_{\text {particulas }}}
$$

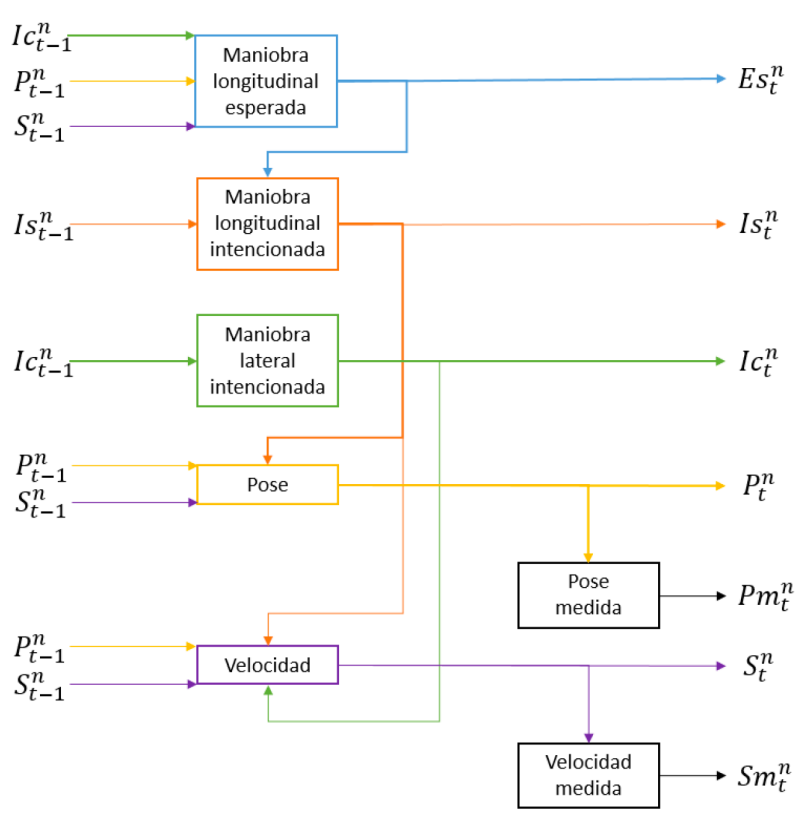

Figura 3: Flujograma del modelo implementado

donde $\mathbf{H}_{\mathbf{i}, \mathbf{t}}$ es el estado $H$ en el instante $t$ de la partícula $i, \mathbf{w}_{\mathbf{i}, \mathbf{t}}$ es el peso $w$ asociado a la partícula $i$ para el mismo instante y $\mathbf{N}_{\text {particulas }}$ corresponde al numero total de partículas empleados en el filtro.

Gracias a su relativa simple implementación, el presente trabajo emplea un filtro secuencial de muestreo por importancia (SIR) [4], también conocido como filtro tipo bootstrap o algoritmo de condensación, cuyas bases de funcionamiento se describen a continuación.

Las variables ocultas que contienen el estado de la partícula se definen con $H_{t}$ y las variables observables (mediciones) como $Z_{t}$. Ambas son conformadas por los siguientes elementos:

$$
\begin{aligned}
H_{t} & =\left(\mathbf{E s}_{\mathbf{t}}, \mathbf{I} \mathbf{c}_{\mathbf{t}}, \mathbf{I s}_{\mathbf{t}}, \mathbf{P}_{\mathbf{t}}, \mathbf{S}_{\mathbf{t}}\right) \\
Z_{t} & =\left(\mathbf{P m}_{\mathbf{t}}, \mathbf{S m}_{\mathbf{t}}\right)
\end{aligned}
$$

Una vez que se tienen las primeras medidas $Z_{0}$ se inicializa el filtro y se sigue la siguiente secuencia:

Predicción: Se calcula el estado de cada partícula en el instante $t$ a partir de su estado en el instante $t-1$ basándose en la función $\pi$ (función de transición):

$$
\begin{aligned}
& \pi\left(H_{t} \mid H_{0: t-1} Z_{0: t}\right)=\prod_{n=1}^{N}\left[P\left(E s_{t}^{n} \mid \mathbf{I}_{\mathbf{t}-\mathbf{1}} \mathbf{P}_{\mathbf{t}-\mathbf{1}} \mathbf{S}_{\mathbf{t}-\mathbf{1}}\right) \times\right. \\
& P\left(I c_{t}^{n} \mid I c_{t-1}^{n}\right) \times P\left(I s_{t}^{n} \mid I s_{t-1}^{n} E s_{t}^{n}\right) \times \\
& \left.P\left(P_{t}^{n} \mid P_{t-1}^{n} S_{t-1}^{n} I c_{t-1}^{n}\right) \times P\left(S_{t}^{n} \mid P_{t-1}^{n} S_{t-1}^{n} I c_{t}^{n} I s_{t}^{n}\right)\right]
\end{aligned}
$$

Actualización: Las medidas en el instante $t$ son usadas para comparar el estado de la partícula 
y verificar si refleja la predicción realizada. La comparación con las medidas se realiza respecto a los pesos $w$ de las partículas:

$w_{i, t} \propto w_{i, t-1} \times \prod_{n=1}^{N} P\left(P m_{t}^{n} \mid P_{t}^{n}\right) \times P\left(S m_{t}^{n} \mid s_{t}^{n}\right)$

Re-muestreo: En función del paso anterior se hace una verificación de la calidad de las muestras. En caso de ser desfavorable, se hace una selección de las partículas y se asignan los mismos pesos para todas. Esto tiene como objetivo evitar degeneración en las muestras, dejando así las partículas cuyos estados reflejen mejor las medidas. Se adopta pues un re-muestreo sistemático segun [4]. El riesgo definido en 2 se puede aproximar mediante la suma de partículas que cumplan con la condición $\left(\left[I s_{t}^{n}=G O\right],\left[E s_{t}^{n}=S T O P\right]\right)$ como se observa a continuación:

$$
\begin{gathered}
P\left(\left[I s_{t}^{n}=G O\right]\left[E s_{t}^{n}=S T O P\right] \mid \mathbf{P m}_{\mathbf{0}: \mathbf{t}} \mathbf{S m}_{\mathbf{0}: \mathbf{t}}\right)= \\
\sum_{i:\left(\left[I s_{t}^{n}=G O\right],\left[E s_{t}^{n}=S T O P\right]\right)} w_{i, t}
\end{gathered}
$$

\subsection{Implementación del filtro}

La solución del filtro de partículas requiere especificar el número de las mismas para ofrecer un buen compromiso entre exactitud de representación de los vehículos y rapidez de cómputo. Para ello se evaluó en la intersección en X el algoritmo detallado en la sección previa usando 200, 400 y 800 partículas, obteniendo los resultados de la tabla 1. De la tabla anterior se extrae que el número

Tabla 1: Horizontes de predicción para los casos de riesgo por número de partículas

\begin{tabular}{|c|c|c|c|c|}
\hline Núm. & Tiempo/ & \multicolumn{3}{|c|}{ Horizontes acum. } \\
\cline { 3 - 5 } partículas & ciclo (s) & $\mathbf{9 0 \%}$ & $\mathbf{5 0 \%}$ & $\mathbf{3 0 \%}$ \\
\hline 200 & 1.04 & 1.95 & 2.13 & 2.18 \\
400 & 2.61 & 2.00 & 2.09 & 2.13 \\
800 & 6.78 & 1.95 & 2.07 & 2.11 \\
\hline
\end{tabular}

de partículas tiene un impacto reducido en el horizonte de predicción. Se puede comprobar que al aumentar el la cantidad, los tiempos para la colisión disminuyen ligeramente para los mismos porcentajes acumulados de detección. Por otro lado, el tiempo por ciclo (TPC) varia notablemente en función del número de partículas. Se tiene que de 200 a 400, el aumento del TPC es de casi el 150 $\%$, mientras que de 400 a 800 partículas el salto es de aproximadamente el $250 \%$.

\section{RESULTADOS}

\subsection{Metodología}

Para generar diferentes escenarios de conducción, tanto desde el punto de visto geométrico como de la dinámica de los agentes que intervienen en ellos, se ha recurrido al simulador de código libre SUMO. Para calificar el desempeño del calculo del riesgo, se evalúan dichos escenarios a partir de los siguientes indicadores:

- Falsas detecciones: Situaciones clasificadas como peligrosas en escenarios donde no hay riesgo.

- Detecciones perdidas: Situaciones no clasificadas como peligrosas en escenarios de riesgo.

- Horizonte de predicción: Tiempo en el cual el modelo puede predecir una maniobra arriesgada. Este horizonte de tiempo $T_{\text {pred }}$ es la diferencia de tiempo que transcurre desde que surge la primera detección $t_{\text {det }}$ (se supera el $\lambda$ establecido) hasta que ocurre la colisión $t_{\text {col }}, T_{\text {pred }}=t_{\text {col }}-t_{\text {det }}$.

Para cubrir un rango significativo de casos, se simularon los tres tipos de intersecciones mas comunes, como son en X, T e Y. Para ello se emplearon diferentes combinaciones de velocidades iniciales y máximas para los vehículos involucrados con el fin de validar el modelo para diferentes escenarios. En la figura 4 se muestran los casos evaluados.
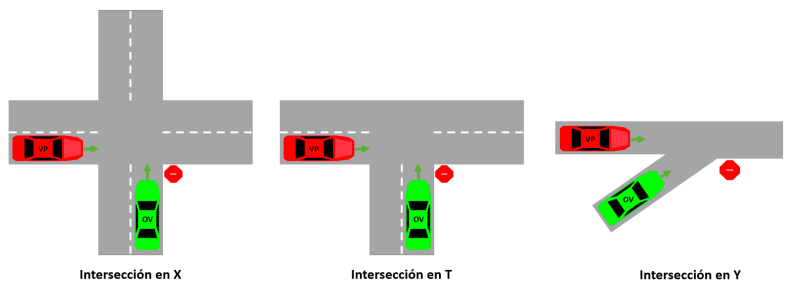

Figura 4: Tipos de intersecciones consideradas

En los dos primeros casos, las vías tienen una longitud de $250 \mathrm{~m}$ hasta el centro de la intersección con un solo carril en ambos sentidos. En los 2 casos las vías horizontales tienen una mayor prioridad -donde circula el vehículo propio (VP)- que las vías verticales donde circula el otro vehículo (OV).

Para todos los vehículos involucrados en la simulación se emplearon los mismos parámetros físicos de aceleración (positiva y negativa), longitud, distancia de seguridad y grado de imperfección, y variaciones sobre las velocidades iniciales y máximas (ver tabla 2) 
Tabla 2: Parámetros físicos de los vehículos

\begin{tabular}{|c|c|}
\hline Aceleración $\left(\mathrm{m} / \mathrm{s}^{2}\right)$ & 1.1 \\
\hline Desaceleración $\left(\mathrm{m} / \mathrm{s}^{2}\right)$ & 4.5 \\
\hline Longitud $(\mathrm{m})$ & 5.0 \\
\hline Distancia mínima $(\mathrm{m})$ & 1.7 \\
\hline Imperfección & 0.2 \\
\hline Rango velocidades $(\mathrm{m} / \mathrm{s})$ & {$[8,14]$} \\
\hline
\end{tabular}

\subsection{Intersección en $\mathrm{Y}$}

En este escenario se tienen dos vehículos: uno prioritario (VP) que cruza la vía de izquierda a derecha y otro $(\mathrm{OV})$ con la intención de unirse a la misma. Para esta única variación del escenario, se comprueban situaciones donde (i) OV se detiene y el riesgo es nulo y (ii) donde existe riesgo de colisión.

El objeto de las simulaciones es determinar si el modelo es capaz de destacar las situaciones peligrosas si estas superan la probabilidad de riesgo $\lambda=0.3$ y evaluar su desempeño con las métricas introducidas en la sección previa. Del total de 120 casos simulados (60 para cada caso), solo una dio falso positivo (tasa de desaciertos del $0.85 \%$ ). En

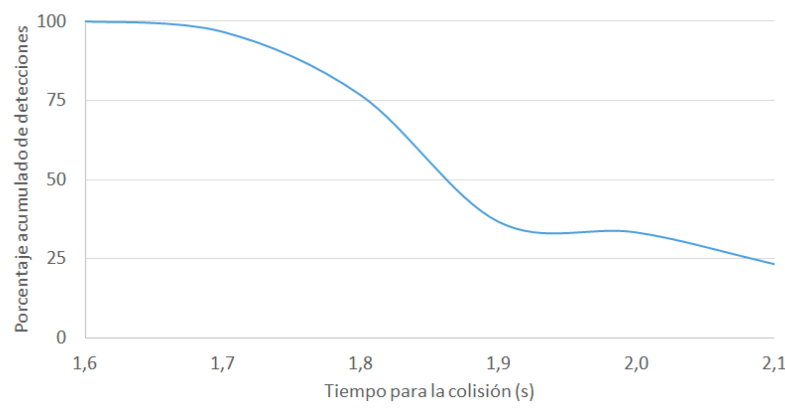

Figura 5: Porcentaje acumulado de detecciones en intersecciones en $\mathrm{Y}$

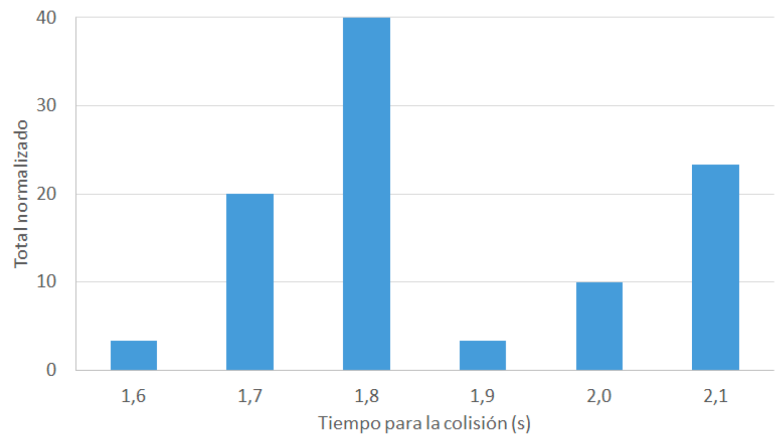

Figura 6: Histograma de detecciones en intersecciones en $\mathrm{Y}$

las Fig. 5 y 6 se observa que el modelo tiene la capacidad de predecir las colisiones hasta con $1.6 \mathrm{~s}$ de antelación como mínimo y aproximadamente el $77 \%$ de las colisiones fueron detectadas por encima de los $1.8 \mathrm{~s}$.

\subsection{Intersección en T}

Nuevamente se simulan dos vehículos aproximándose a una intersección por dos de los tres frentes posibles. El vehículo que atraviesa la vía de izquierda a derecha (VP) tiene prioridad sobre el vehículo que ingresa en la parte inferior y la abandona o bien por la derecha como la izquierda (OV). Se contemplan dos casos: donde OV obedece (no hay riesgo) o no a la señal de Stop (escenario de riesgo). Para este escenario se realizaron un total de 240 simulaciones considerando esos 2 casos de riesgo y una combinación de diferentes perfiles de velocidad y rutas. De las 120 simulaciones

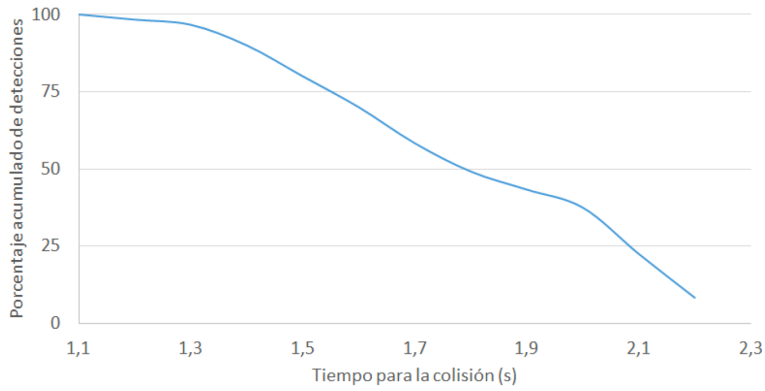

Figura 7: Porcentaje acumulado de detecciones en intersecciones en $\mathrm{T}$

realizadas para los casos donde el riesgo es nulo se extrajeron 5 falsos positivos, lo que implica una tasa de desaciertos del $4.2 \%$. Por otro lado, el 100\% de los casos de riesgo fueron reconocidos. Para los casos de riesgo, las figuras 7 y 8 indican que aproximadamente el $50 \%$ de las maniobras peligrosas se detectaron por encima de $1.8 \mathrm{~s}$ antes de la colisión. De manera similar, se extrae que el modelo es capaz de predecir una maniobra de riesgo con una antelación mínima de 1.1s.

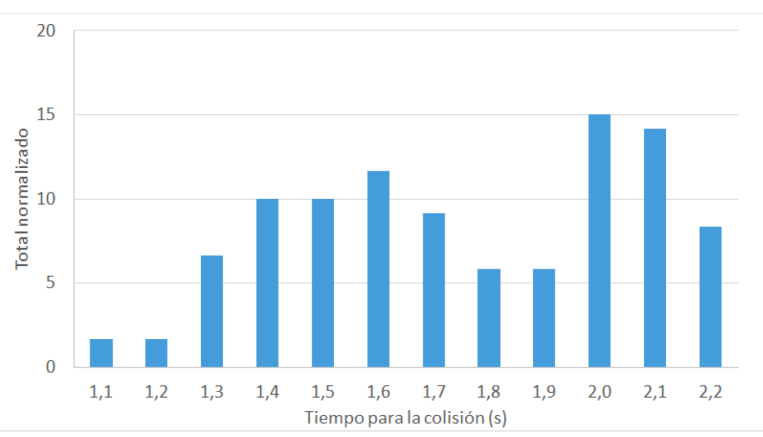

Figura 8: Histograma de detecciones en intersecciones en $\mathrm{T}$ 


\subsection{Intersección en X}

Para este caso se simulan dos vehículos aproximándose a una intersección en $\mathrm{X}$, teniendo VP prioridad sobre OV. De nuevo mientras VP atraviesa la vía de izquierda a derecha, se comprobarán las tres posibles rutas que puede tomar OV al negociar con la intersección con los velocidades mencionados anteriormente. Se realizaron un

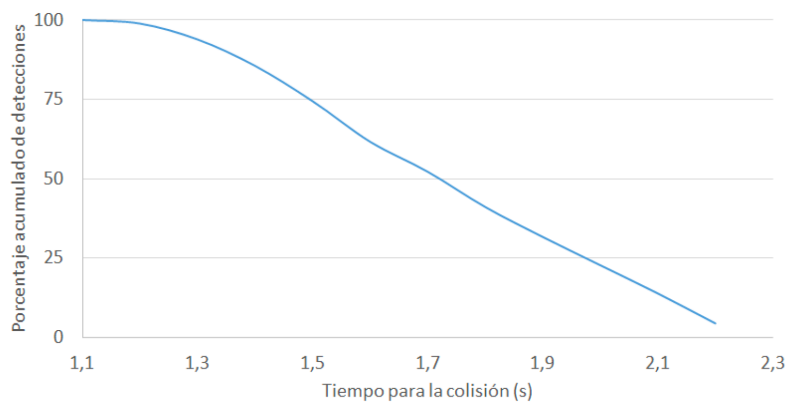

Figura 9: Porcentaje acumulado de detecciones en intersecciones en $\mathrm{X}$

total de 360 simulaciones, de entre las que las 180 simulaciones del caso de riesgo presentaron. 6 falsos positivos y Para los casos de riesgo, el $100 \%$ de las colisiones fueron detectadas (1.7\% tasa de desacierto total). La Fig. 9 muestran que para los rangos de velocidad probados, el tiempo previo a una colisión mas bajo fue de 1.1s, y casi el $75 \%$ de todos los casos fueron detectados sobre $1.5 \mathrm{~s}$ antes de la colisión.

\subsection{Evaluación con más de dos vehículos}

Para probar la aplicación del modelo en escenarios mas realistas, se comprueba su funcionamiento con tres vehículos aproximándose a una intersección en Y. De manera similar a la Sec. 5.2, se tienen dos vehículos (ahora VP1 y VP2) con prioridad sobre OV. Estos atraviesan la vía mientras OV tiene que negociar con la intersección y detenerse o no para ceder el paso. Se ejecutan las pruebas sincronizando a los vehículos para inducir un contacto en caso de que no se detengan. Los vehículos VP1 y VP2 circulan por la vía con una distancia de separación de 10 metros y se evalúan dos casos, uno donde OV se sincroniza con VP1 y el otro con VP2. Se realizaron 240 simulaciones, de entre las cuales 60 reprodujeron casos de riesgo con OV entrando en contacto con VP1 y otras 60 con VP2. En todas ellas la tasa de acierto fue del 100\%. De las 120 simulaciones correspondientes a los casos de riesgo, las colisiones fueron detectadas con una antelación mínima de 1.6s. Según las figuras 10 y 11 en ambos casos cerca del $85 \%$ acumulado de las detecciones se sitúan en alrededor de los 1.9s previos a la colisión. En definitiva,

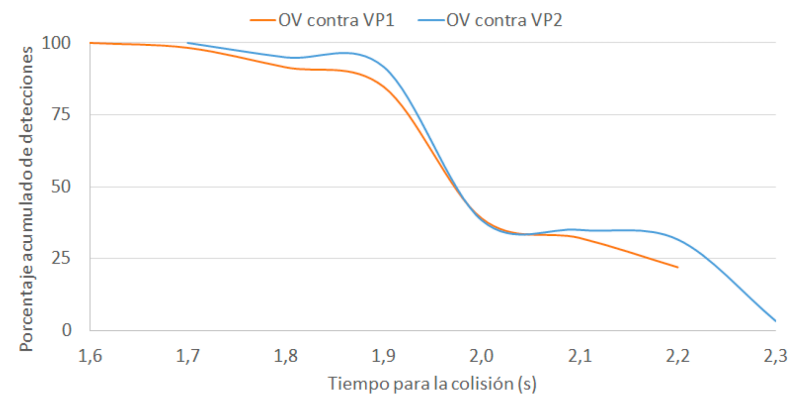

Figura 10: Histograma de detecciones con más de 2 vehículos

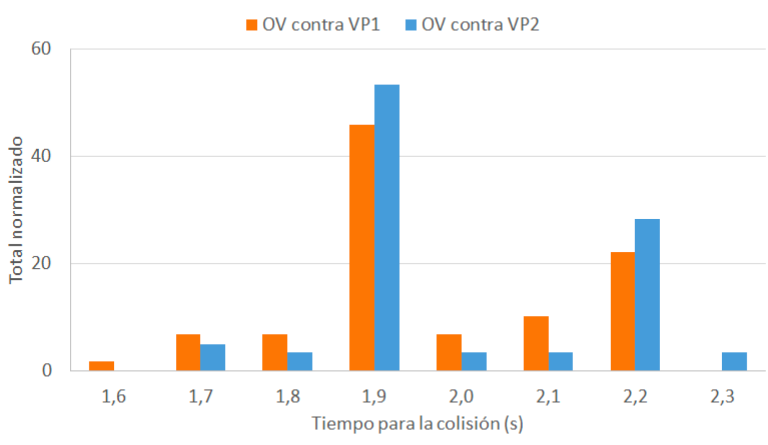

Figura 11: Histograma de detecciones con más de 2 vehículos

se comprueba la capacidad del modelo para determinar con una antelación razonable el riesgo en el contexto de cada situación. Se demuestra igualmente que la estructura del modelo puede ser adaptada a condiciones reales de tráfico, tanto en la topología de la intersección como en el numero de vehículos. Es reseñable sin embargo que al aumentar el numero de vías que componen la intersección, los porcentajes de tiempos para la colisión detectados se uniformizan.

\section{CONCLUSIONES}

El articulo presenta una estrategia de estimación de riesgos que tiene en cuenta tanto el contexto topológico de la escena de conducción como la interacción entre los agentes. El filtro de partículas sobre el cual repose la inferencia de la estimación de la intención y la expectativa se ha evolucionado, respecto a una versión precedente, con objeto de adaptarlo a cada una de las situaciones evaluadas. Se ha conseguido validar mediante simulaciones en 3 tipos de intersecciones el modelo sobre el que reposa el filtro, que es capaz de anticipar con una alta tasa de éxito los casos en los que hay riesgo de realizar una maniobra con riesgo de colisión entre dos vehículos. Antes de su implementación sobre vehículo real, se introducirá un modelo de expectativa lateral y se evaluará su comportamiento con 
un conjunto elevado de vehículos en situaciones de mayor complejidad.

\section{Agradecimientos}

Los autores quieren agradecer la ayuda financiera recibida del proyecto COGDRIVE (DPI201786915-C3-1-R)

\section{English summary}

VALIDATION OF A STRATEGY
FOR RISK ESTIMATION AT
INTERSECTIONS WITH CON-
NECTED VEHICLES

\section{Abstract}

To make possible the massive deployment of automated vehicles in urban environments, it is essential to move forward in making safe decisions. In particular, it is necessary to improve the ability to infer the intentions of the different agents and the risk involved in complex driving scenes, thus improving the safety and predictability of automated driving and driving assistance systems. The present work shows the implementation and validation in simulation of a novel solution to estimate the risk of driving using a model of space and states in the context of intersections. The strategy used models the driving scene as a dynamic Bayesian network and infers intentions and expectations of the agents involved through a particle filter. The results are very promising in both the success rate and the prediction horizon in the environments for which it has been tested: $Y, T$ and $X$ intersections.

Keywords: Risk assessment, Intelligent vehicles, Dynamic Bayesian Network, Particle Filter

\section{Referencias}

[1] M. Althoff and A. Mergel. Comparison of markov chain abstraction and monte carlo simulation for the safety assessment of autonomous cars. IEEE Transactions on Intelligent Transportation Systems, 12(4):12371247, 2011.
[2] G. S. Aoude et al. Threat assessment design for driver assistance system at intersections. In Proc. IEEE Intelligent Transportation Systems (ITSC), pages 1855-1862, 2010.

[3] G. S. Aoude et al. Driver behavior classification at intersections and validation on large naturalistic data set. IEEE Transactions on Intelligent Transportation Systems, 13(2):724-736, 2012.

[4] Arulampalam et al. A tutorial on particle filters for online nonlinear/non-gaussian bayesian tracking. IEEE Transactions on signal processing, 50(2):174-188, 2002.

[5] M. Brand, N. Oliver, and A. Pentland. Coupled hidden markov models for complex action recognition. In Proc. Comp. Vision and Pattern Recognition, pages 994-999, 1997.

[6] S. Lefèvre, C. Laugier, and J. IbañezGuzmán. Risk assessment at road intersections: Comparing intention and expectation. In IEEE Intelligent Vehicles Symposium (IV), pages 165-171, 2012.

[7] S. Lefèvre, D. Vasquez, and C. Laugier. A survey on motion prediction and risk assessment for intelligent vehicles. Robomech Journal, 1(1):1, 2014.

[8] M. Liebner et al. Driver intent inference at urban intersections using the intelligent driver model. In IEEE Intelligent Vehicles Symposium (IV), pages 1162-1167, 2012.

[9] C.-F. Lin et al. Vehicle dynamics and external disturbance estimation for vehicle path prediction. IEEE Transactions on Control Systems Technology, 8(3):508-518, 2000.

[10] K. P. Murphy and S. Russell. Dynamic bayesian networks: representation, inference and learning. 2002.

[11] M. G. Ortiz et al. Behavior prediction at multiple time-scales in inner-city scenarios. In IEEE Intelligent Vehicles Symposium (IV), pages 1068-1073, 2011.

[12] A. Spek, P. A. Wieringa, and W. Janssen. Intersection approach speed and accident probability. Transportation Research Part F: Traffic Psychology and Behaviour, 9(2):155171, 2006.

[13] C. Tay. Analysis of dynamic scenes: application to driving assistance. L'Institut Poly technique de Grenoble, FranceDèpt. Telecommun., 2009. 